\title{
Estratégias de coping em profissionais da saúde durante a pandemia em um hospital
}

\section{na Amazônia legal}

\author{
Coping strategies in health professionals during the pandemic in a hospital in the legal Amazon \\ Enfrentando estrategias en profesionales de la salud durante la pandemia en un hospital de la
}

Amazonia legal

Recebido: 24/09/2021 | Revisado: 30/09/2021 | Aceito: 14/10/2021 | Publicado: 17/10/2021

\author{
Thainá Sampaio Ribeiro ${ }^{1}$ \\ ORCID: https://orcid.org/0000-0001-7344-8517 \\ Hospital Regional de Cacoal, Brasil \\ E-mail: thaina_sampaio@ hotmail.com \\ Nádia Maria Silva Montelo \\ ORCID: https://orcid.org/0000-0003-1525-4954 \\ Hospital Regional de Cacoal, Brasil \\ Faculdade de Ciências Biomédicas de Cacoal, Brasil \\ E-mail: Nadia_montello@hotmail.com \\ Sheila Carminati de Lima Soares ${ }^{3}$ \\ ORCID: https://orcid.org/0000-0002-8219-9716 \\ Faculdade de Ciências Biomédicas de Cacoal, Brasil \\ Hospital Regional de Cacoal, Brasil \\ E-mail: Shecarminati@yahoo.com.br
}

\begin{abstract}
Resumo
Introdução - O presente estudo investigou e apresenta as Estratégias de Coping mais utilizadas por profissionais da saúde de um hospital localizado no interior da Amazônia Legal, que foi adaptado para ser referência frente à pandemia ocasionada pelo novo Coronavírus. Objetivos - Buscou-se identificar e descrever as estratégias de enfrentamento relacionadas à atuação de profissionais da saúde no combate à pandemia da Covid-19. Métodos Trata-se de um estudo qualitativo, descritivo e exploratório, desenvolvido com 100 profissionais da saúde atuantes em um hospital referência a pacientes graves durante a pandemia. Métodos. Os dados foram coletados de Março a Abril de 2021, mediante a entrevista estruturada e aplicação da escala Toulousiana de coping, sendo utilizada para análise de dados a tabulação via Excel e demonstrado através de um gráfico para maior clarificação dos resultados. Resultados - Notou-se uma prevalência em relação à estratégia de enfrentamento de controle entre os profissionais entrevistados. Acredita-se que o controle está ligado a pratica profissional e a tomada de decisão imediata presente constantemente entre os profissionais que atuam na saúde, essas estratégias de enfrentamento são utilizadas como um recurso a fim de minimizar o sofrimento inerente às mudanças e adaptações cotidianas. Conclusão - faz-se necessário realizar mais estudos em relação aos aspectos emocionais e comportamentais experienciado por profissionais da saúde, apesar do aumento na produção de dados que evidenciem características regionais, os mesmos ainda são incipientes para retratar a realidade.
\end{abstract}

Palavras-chave: Coping; Pandemia; Profissionais de saúde.

\begin{abstract}
Introduction - The present study investigated and presents the Coping Strategies most used by health professionals of a hospital located in the interior of the Legal Amazon, which was adapted to be a reference against the pandemic caused by the new Coronavirus. Objectives - We sought to identify and describe the coping strategies related to the performance of health professionals in fighting the Covid-19 pandemic. Methods - This is a qualitative, descriptive and exploratory study, developed with 100 health professionals working in a reference hospital for critically ill patients during the pandemic. Methods. The data were collected from March to April 2021, through a structured interview and application of the Toulousian coping scale. Results - We noticed a prevalence in relation to the coping
\end{abstract}

\footnotetext{
${ }^{1}$ Psicóloga, Especialista em Saúde mental, Pós graduanda em terapia intensiva através do programa de residência em cuidados intensivos do Hospital Regional de Cacoal (HRC). Brasil.

${ }^{2}$ Psicóloga, especialista em Psicologia Social. Atua como psicóloga no Hospital Regional de Cacoal (HRC) é tutora do programa de residência multiprofissional e docente na Faculdade de Ciências Biomédicas de Cacoal (UNIFACIMED) Brasil.

${ }^{3}$ Enfermeira, mestre em Ciências da Saúde, Docente na Faculdade de Ciências Biomédicas de Cacoal (UNIFACIMED) e enfermeira do Hospital Regional de Cacoal (HRC) Brasil.
} 
strategy of control among the professionals interviewed. It is believed that control is linked to professional practice and immediate decision making, which is constantly present among health professionals. These coping strategies are used as a resource to minimize the suffering inherent to daily changes and adaptations. Conclusion - it is necessary to conduct further studies regarding the emotional and behavioral aspects experienced by health professionals, despite the increase in the production of data that show regional characteristics, they are still incipient to portray the reality.

Keywords: Coping; Pandemic; Health professionals.

\section{Resumen}

Introducción - El presente estudio investigó y presenta las Estrategias de Enfrentamiento más utilizadas por los profesionales de la salud de un hospital ubicado en el interior de la Amazonia Legal, que fue adaptado para ser una referencia contra la pandemia causada por el nuevo Coronavirus. Objetivos - Se busca identificar y descifrar las estrategias de afrontamiento relacionadas con la actuación de los profesionales de la salud en la lucha contra la pandemia del Covid-19. Métodos - Se trata de un estudio cualitativo, descriptivo y exploratorio, desarrollado con 100 profesionales de la salud en un hospital de referencia para pacientes graves durante la pandemia. Métodos. Los datos fueron recogidos de marzo a abril de 2021, a través de la entrevista estructurada y la aplicación de la escala de afrontamiento de Toulousian, siendo utilizado para el análisis de datos la tabulación a través de Excel y se muestra a través de un gráfico para una mayor aclaración de los resultados. Resultados - Se observó una prevalencia en relación con la estrategia de afrontamiento de control entre los profesionales entrevistados. Se cree que el control está vinculado a la práctica profesional y a la toma de decisiones inmediatas, que está presente constantemente entre los profesionales de la salud. Estas estrategias de afrontamiento se utilizan como recurso para minimizar el sufrimiento inherente a los cambios y adaptaciones diarias. Conclusión - es necesario realizar más estudios en relación a los aspectos emocionales y conductuales vividos por los profesionales de la salud, a pesar del aumento en la producción de datos que muestran las características regionales, los mismos son aún incipientes para retratar la realidad.

Palabras clave: Afrontamiento; Pandemia; Profesionales de la salud.

\section{Introdução}

Individualmente o ser humano se organiza e realiza planejamentos a curto, médio e longo prazo, definindo ações para o alcance dos objetivos idealizados. No entanto, diariamente se depara com novas situações que, por muitas vezes, trazem mudanças ao que previamente havia sido pensado. Isso exige que, frente às novas configurações, haja uma resposta ou a utilização de algum recurso para adaptação, podendo ser considerados como positivos ou negativos.

A necessidade de lidar com a nova configuração e a impossibilidade da realização do que havia sido planejado influencia aspectos da saúde física e mental. Segundo a Organização Mundial da Saúde (OMS), saúde mental refere-se ao bem estar biopsicossocial, envolvendo a capacidade de reconhecer suas habilidades e manejar suas limitações. Ao longo do desenvolvimento humano, as pessoas constroem habilidades como recursos de enfrentamento para lidarem com situações de crise.

Em dezembro de 2019, o mundo presenciou o início de uma grande mudança no contexto de saúde, surgindo os primeiros casos de contaminação por Covid-19, fato esse que trouxe impactos biológicos, psicológicos e sociais. Diante disso, vale citar as mudanças abruptas nas rotinas de trabalho, interação social, higiene pessoal e diversos comportamentos responsáveis para a prevenção e não contaminação da doença (Opas, 2020).

Muitas pessoas tiveram a opção de desenvolver suas rotinas diárias de maneira remota, conseguiram diminuir o contato com o outro e construíram barreiras de distanciamento social. Dentro de o ambiente hospitalar também houve alterações significativas, porém, como se constitui um serviço essencial, a possibilidade de ficar em casa, não se configurou como uma opção válida aos profissionais de saúde (Opas, 2020).

Dentro do Sistema Único de Saúde (SUS), como estratégia para lidar com o momento de pandemia, foram destinados dentro das unidades hospitalares leitos para o tratamento das formas graves da infecção. Foram também criados novos leitos e abertas unidades que foram denominadas "hospitais de campanha". Para tanto, foi adquirido toda uma estrutura para a organização dessas unidades, que vão desde os equipamentos para esses novos leitos a insumos para garantir o funcionamento dos mesmos (Brasil, 2020). 
Para que toda essa estrutura pudesse funcionar, o fator indispensável é o ser humano, sendo nesse caso especificamente os profissionais de saúde. Assim como o número de leitos, o quantitativo de profissionais nas unidades de saúde necessitou também ser aumentado. Reorganizações nos ambientes de trabalho foram necessárias onde se aumentou o número de leitos nas unidades, porém diminuiu-se o número de profissionais nesses ambientes. Para intervir nessa problemática foram criadas inúmeras vagas de trabalho para profissionais de saúde (Teixeira et al, 2020).

Para minimizar o déficit de recursos humanos o Governo do Estado de Rondônia contratou, via processo seletivo para contrato temporário, profissionais para complementar as escalas de trabalho, assim se formaram as equipes de trabalho para atender as demandas da população referenciada ao Hospital Regional de Cacoal (HRC).

A atuação dos profissionais de saúde é delicada, pois lida com extremos e as possibilidades de morte/vida. A prática fomenta experiências de perdas, estresse, ansiedade, aspectos que podem afetar a vida psicológica desses profissionais. Logo, se torna necessário cuidar da saúde mental a fim de prevenir ou diminuir o sofrimento psíquico desses indivíduos (Teixeira $E t$ $a l, 2020)$.

Diante do exposto, o objetivo da pesquisa foi identificar e descrever as estratégias de enfrentamento relacionadas à atuação de profissionais da saúde no combate à pandemia da Covid-19. O levantamento dessas informações torna-se pertinente, pois fomenta iniciativas e novas pesquisas que elucidem aspectos próprios da região Norte, sendo de suma importância para dar-lhes visibilidade aos aspectos da saúde mental, como também no intuito de contribuir com outros estudos

\section{Covid-19}

No final do ano de 2019, especificamente no dia 31 de dezembro, em Wuhan, na China, foi detectado o primeiro caso de SARS-CoV-2, considerado o causador da doença Covid-19. O SARS-CoV é vírus causador da síndrome respiratória aguda grave ou SARS. O quadro inicial da Covid-19 é característico de uma síndrome gripal, porém, o quadro clínico de infecção pelo coronavírus é amplo, podendo variar de sintomas leves como, resfriados até uma pneumonia grave (Lima, 2020).

Em 9 de janeiro de 2020 a Organização Mundial da Saúde (OMS) confirmou a circulação do novo coronavírus. Durante o mês de janeiro, diversos países apresentavam casos confirmados do novo Coronavírus, incluindo Estados Unidos, Canadá e Austrália e a velocidade de propagação chamou atenção devido ao número médio de casos identificados (Lana Et al, 2020).

No dia 30 de janeiro de 2020, após ocasionar as primeiras mortes e se espalhar rapidamente em escala mundial, a Organização Mundial de Saúde (OMS) declarou emergência de saúde pública de importância Internacional e no Brasil através da portaria nº 188/GM/MS, de 03 de fevereiro de 2020, foi declarado situação de emergência Nacional, em decorrência da contaminação por coronavírus (Brasil, 2020). Em sequência, a Lei nº 13.979, de 06 de fevereiro de 2020, estabeleceu as medidas para enfrentamento de emergência em saúde pública de importância internacional decorrente do coronavírus (Covid19) e cita aspectos visando à proteção da coletividade. Assim, diante do contexto de pandemia, houve uma mudança no cenário nacional, paralisação dos comércios, empresas liberaram os funcionários, foi decretado isolamento social (Brasil, 2020).

A contaminação através da Covid-19 desencadeou uma pandemia, trazendo alterações nas rotinas e estilos de vida, como, períodos de quarentena, distanciamento social, serviços de home care, mesmo com as diversas medidas, houve grande número de contaminados e um aumento significativo no número de internações e óbitos decorrentes da doença, indicando um colapso no Sistema de Saúde (Brasil, 2020).

Em todo o território Nacional houve decretos de calamidade pública, o cenário tornou-se assustador, houve a criação de novos leitos para internação hospitalar, hospitais de campanha, contração emergencial de recursos humanos, diversas foram às adaptações a fim de oferecer cuidados no âmbito da saúde. Entretanto as adaptações não foram suficientes para atender o 
número de contaminados, assim a população de maneira geral passou a vivenciar as angústias inerentes à falta de uma medicações e vacinas que auxiliassem na contenção da doença, dessa forma, o cenário de crise se fortalecia (Opas, 2020).

\section{Práxis - Emergências e Desastres}

Diversos fenômenos têm afetado a vida cotidiana das pessoas, a citar: eventos climáticos ou tecnológicos, controláveis, evitáveis ou não. Tais eventos são desencadeadores de danos psicológicos aos indivíduos (Franco, 2015). De acordo com a Jacob e Cibim (2015) os desastres são caracterizados como eventos que acarretam prejuízos nas esferas biológicas, psicológicas e sociais, a considerar a perda de vidas humanas, prejuízos na saúde pública, nas propriedades ou até mesmo ambientais. E para lidar com as vivências diante de um desastre é importante avaliar a subjetividade da comunidade visando às necessidades específicas, objetivas e as possibilidades de atuação (Paulino \& Sant’Ana, 2018).

No caso da pandemia ocasionada pelo novo coronavírus, todos foram afetados direta ou indiretamente, no caso dos profissionais da saúde o trabalho desenvolvido por eles em situações de crise os expõe a diversas reações negativas, pressão sobre as atitudes e decisões que terão que tomar, além de lidar com a intensidade de um ambiente estressor. Os profissionais colocam em risco sua saúde física e mental. O período de exposição pode trazer consequências para quem trabalha em situações de crises e emergências, podendo desenvolver, síndrome de estresse pós-traumático (TEP), burnout, síndrome do esgotamento profissional, exaustão emocional, despersonalização e baixo comprometimento, tais consequências são perceptíveis a longos períodos (Franco, 2013).

Diante da vivência é importante refletir sobre as estratégias de autocuidado que podem ser realizadas no momento de emergências e desastres a fim de ressignificar as novas condições de vida e as perspectivas de futuro. A intervenção psicológica de emergência deve ser ofertada em todo o mundo, evidenciando a importância dos cuidados com a saúde mental (Franco, 2015; Paulino e Sant’Ana, 2018).

\section{Estratégias de Coping}

Ao longo do desenvolvimento humano são vivenciadas diversas situações, sejam elas de aspectos positivos ou negativos. Tais vivências costumam gerar crescimento e desenvolvimento pessoal. Ao se deparar com situações que são estressoras e desgastantes e no intuito de autopreservação, os indivíduos se utilizam de artifícios cognitivos e comportamentais como forma de enfrentamento à situação, a esses artifícios dá-se o nome de coping. Na língua portuguesa, essa palavra não apresenta tradução. Porém, muitos autores utilizam o termo enfrentamento, isto é, estratégias utilizadas de forma consciente para lidar com o problema (Lazarus \& Folkman, 1984).

Coping é conceituado como conjunto de determinantes estratégicos que engloba o nível cognitivo e comportamental, com o objetivo de minimizar o sofrimento vivenciado. As estratégias de coping são aprendidas e desenvolvidas ao longo da vida. Desta forma, as estratégias utilizadas variam de acordo com a subjetividade do sujeito, a considerar, sexo, idade, contexto social, história do desenvolvimento e o repertório cognitivo comportamental (Nunes, et al., 2014).

Em geral pode-se dizer que estratégia de enfrentamento é um conjunto de estratégias utilizadas por pessoas para lidarem com as situações, circunstâncias adversas, sendo avaliado como um momento transicional entre o sujeito e o ambiente, é considerado um objeto de estudo da psicologia clínica, social e da personalidade (Fernandes e Inocente, 2011).

Lazarus e Folkman (1984) definem enfrentamento como conjunto de esforços cognitivos e comportamentais em constante mudança, através das estratégias desenvolvidas e o repertório comportamental, o indivíduo consegue minimizar, tolerar, aceitar e controlar situações e necessidades especificas. 
Percebe-se uma subdivisão nas estratégias de enfrentamento, sendo padrões diretos, nos quais se refere ao uso de habilidades para solucionar o problema e padrões indiretos, estratégias que não modificam as demandas, mas altera a forma como as pessoas experimentam a demanda (Fernandes e Inocente, 2011).

Segundo Lazarus \& Folkman (1984), o coping caracteriza-se por um conjunto de recursos cognitivos e comportamentos utilizados para manejar demandas, internas ou externas que geram sobrecarga nas relações interpessoais. Os autores dividem as estratégias em dois grupos: (1) centrada sobre o problema, (2) centrada sobre as emoções, podendo ser utilizadas ao mesmo tempo diante da avaliação da situação.

Em alguns momentos as pessoas se deparam com situações que evidenciam as particularidades de cada indivíduo: biológica, psicológica e social. O ser humano é um ser adaptativo, desta forma diante das situações vivenciadas, o mesmo emite um conjunto de respostas às situações que se modificam e, consequentemente, o organismo desenvolve uma estratégia satisfatória. (Sandeiro Et al., 2016).

As estratégias de coping são utilizadas para reduzir o nível de stress, desenvolvendo reações físicas ou emocionais. Ao longo dos anos, vários estudos foram desenvolvidos para identificar, analisar e compreender as estratégias utilizadas, algumas escalas foram desenvolvidas para classificar a resposta do sujeito.

\section{Material e Método}

\section{Escala Toulousiana de Coping}

A escala utilizada na pesquisa foi a Toulousiana de coping (1993) versão (2014) que é organizada em campos: comportamental - ligado à ação do indivíduo; cognitivo - que transparece o acesso à informação; e afetivo - evidenciando as emoções presentes na vivência.

A escala é composta por dezoito itens, distribuídos em 5 (cinco) fatores estratégicos: (1) Controle, (2) Recusa, (3) Conversão, (4) Suporte Social e (5) Distração. Os dados foram avaliados pelo próprio indivíduo numa escala de cinco pontos: 1- "nunca"; 5- "muita frequência". O score individual é calculado mediante a média de cada fator (Nunes, et al. 2014).

\section{Método}

A pesquisa foi realizada no município de Cacoal- RO, o procedimento de coleta de dados teve início após aprovação do projeto de pesquisa pelo Comitê de Ética em Pesquisa (CEP) sob o número de parecer 4.619.62, conforme Resolução 466/12, e posterior autorização da direção da instituição hospitalar para o desenvolvimento da mesma por intermédio da assinatura da carta de anuência.

Gil (2008, p.89) cita amostra como uma parcela da população, característica e representativa da população que se pretende estudar. A população envolvida constitui-se de uma amostragem intencional e por conveniência.

O estudo foi realizado utilizando-se os tipos de pesquisa quantitativa e qualitativa. Neste tipo de pesquisa, "[...] as limitações de um método podem ser compensadas pelas potencialidades do outro método, e a combinação de dados quantitativos e qualitativos proporciona um entendimento mais completo do problema da pesquisa do que cada uma das abordagens isoladamente" (Creswell; Plano Clark, 2013, p.25).

A pesquisa realizada de punha quantitativo que evidencia os resultados, através dos dados numéricos avaliados. Esse método gera dados que podem ser demonstrados através de porcentagens, estatísticas e probabilidades. (Pereira A. S. et al. 2018)

Os participantes da pesquisa foram profissionais que compõem as mais diversas equipes multiprofissionais de saúde da instituição, sendo exclusivamente servidores do Hospital, com os mais diferentes vínculos (estatutários, emergenciais e 
terceirizados) e também os profissionais inseridos na instituição pelo Programa de Residência Multiprofissional em Saúde (PRMS).

Quando foi realizada a aplicação do questionário o quadro de funcionários da equipe multiprofissional atuantes na linha de frente da Covid-19 era de 211 (duzentos e onze) servidores assistenciais, conforme informação repassada pela equipe gestora da Instituição. A pesquisa foi validada a partir da participação de profissionais que compõem a equipe multiprofissional, foram entrevistados, durante o período de Março a Abril de 2021, 100 pessoas, resultado do cálculo da média dos profissionais da saúde atuantes na linha de frente, a considerar o erro amostral de 5\%.

Para alcance dos objetivos foram utilizados dois instrumentos para coleta de dados, sendo um questionário sociodemográfico, a fim de abordar aspectos pessoais e profissionais, e a escala Echelle Toulousaine de Coping versão (2014), considerada uma ferramenta científica para avaliar as estratégias de enfrentamento.

A participação na pesquisa aconteceu após manifestação de aceite por meio da assinatura do Termo de Consentimento Livre e Esclarecido pelo participante, assim, sendo solicitado o preenchimento do questionário sociodemográfico e posteriormente o servidor foi direcionado ao preenchimento da escala de Echelle Toulousiane de Coping, versão (2014). Todas as entrevistas foram realizadas individualmente, respeitando o distanciamento social, a fim de estimular a coleta de dados, garantindo assim, a privacidade e sigilo, além de manter os cuidados com a saúde física.

A análise de dados foi realizada através da tabulação e cálculos via Excel, resultando em dados numéricos absolutos e relativos, assim, evidenciando as principais estratégias de enfrentamento utilizadas por profissionais de saúde no contexto de pandemia, através destes mesmos dados foi elaborado um gráfico para maior clarificação dos resultados obtidos.

\section{Resultados e Discussões}

\section{Caracterização da amostra}

A amostra da pesquisa foi composta por profissionais da saúde, servidores públicos que trabalham no Hospital Regional de Cacoal, sendo 100 (cem) participantes. Desses, foram validados 83 (oitenta e três), o restante foi excluído por questionários incompletos.

Entre os participantes, $71,42 \%$ foram do gênero feminino, $28.57 \%$ do gênero masculino. Os voluntários apresentam variantes de idade, entre 50 (cinquenta) e 22 (vinte e dois) anos, sendo a média de 33.62 (trinta e três) anos de idade. Entre os participantes, $51,64 \%$ são casados, $39.56 \%$ são solteiros, $8.79 \%$ são divorciados. Em aspectos similares, numa pesquisa realizada por Chamon (2006), 80 (oitenta) profissionais da saúde, apresentou dados convergentes em relação à média de idade dos participantes, 34 (trinta e quatro) anos. Em contrapartida numa pesquisa realizada por Da Silva Et al (2017), a média de idade foi de 39 (trinta e nove anos).

A pesquisa contou com profissionais em diferentes níveis de formação acadêmica, desde ensino médio técnico à especialização. Entre os profissionais participaram médicos, enfermeiros, nutricionistas, psicólogos, assistentes sociais, farmacêuticos, técnicos de enfermagem, técnicos em nutrição, fisioterapeutas e agentes administrativos, profissionais esses atuantes nas unidades destinadas a pacientes com Covid-19.

Os participantes relataram diferentes vínculos empregatícios, 37.36\% são estatutários, profissionais concursados efetivos no hospital, 51.64\% são profissionais de contrato temporário, denominados como contratação emergencial e $12.08 \%$ são profissionais pós-graduandos através do programa de residência multiprofissional em cuidados intensivos.

O tempo de serviço profissional na instituição, apresenta variável entre três e seis meses o que corresponde a 7.69\%, de seis meses há 01 ano $46.15 \%$, entre 01 a 05 anos de serviço $26.36 \%$, e acima de 05 anos de serviço, profissionais excepcionalmente estatutários correspondem a 21.97\%. O resultado apresentado vai de encontro com o Da Silva eal (2017) que 
em sua pesquisa, a amostra validada com o tempo mínimo de serviço foi de seis meses. Já na pesquisa realizada por Chamon (2006), os profissionais entrevistados trabalhavam em diversos setores, no tempo mínimo de serviço em 01 ano de atuação.

Entre os aspectos abordados através do questionário sociodemográfico, questionou-se sobre o acompanhamento psicológico, $78 \%$ dos participantes declararam não realizar acompanhamento. Esse aspecto não foi abordado em pesquisas encontradas e anteriormente realizadas.

A seguir apresentaremos em tabela os dados obtidos no questionário sociodemográfico, especificando a divisão da amostra total com base nos itens: gênero, estado civil, formação, vinculo, tempo de serviço.

Tabela 1: Distribuição dos dados sociodemográficos dos profissionais de saúde atuantes na linha de frente do Covid-19 no período de Março a Abril no Hospital Regional de Cacoal. Cacoal/RO, 2021.

\begin{tabular}{|c|c|c|}
\hline Itens Avaliados & N (Total) & n (\%) \\
\hline \multicolumn{3}{|l|}{ Gênero } \\
\hline Masculino & 24 & $28.57 \%$ \\
\hline Feminino & 59 & $71.42 \%$ \\
\hline \multicolumn{3}{|l|}{ Estado civil } \\
\hline Solteiro & 07 & $8.79 \%$ \\
\hline Casado/ união estável & 43 & $51,64 \%$ \\
\hline Divorciado/ separado & 33 & $39.56 \%$ \\
\hline Viúvo & 00 & $0 \%$ \\
\hline \multicolumn{3}{|l|}{ Nível de formação } \\
\hline Ensino médio & 18 & $21.68 \%$ \\
\hline Ensino superior & 27 & $32,53 \%$ \\
\hline Especialização & 37 & $44,57 \%$ \\
\hline \multicolumn{3}{|l|}{ Vinculo } \\
\hline Estatutário & 31 & $37.36 \%$ \\
\hline Residente & 10 & $12.08 \%$ \\
\hline Emergencial & 42 & $51.64 \%$ \\
\hline \multicolumn{3}{|l|}{ Tempo de serviço } \\
\hline 03 a 06 meses & 06 & $7.69 \%$ \\
\hline 06 meses a 1 ano & 38 & $46.15 \%$ \\
\hline 1 a 5 anos & 21 & $26.36 \%$ \\
\hline 5 a 10 anos & 18 & $21.97 \%$ \\
\hline
\end{tabular}

Fonte: Ribeiro, Montelo \& Soares (2021).

\section{Estratégias de enfrentamento utilizadas pelos profissionais de saúde}

Os resultados encontrados reúnem aspectos emocionais, psicológicos e comportamentais, que os profissionais da saúde utilizam frente à realidade que estão inseridos. A escala se divide em alguns aspectos, sendo eles: recusa, suporte social, conversão, distração e controle. 
A seguir apresenta-se o gráfico que demonstra os resultados encontrados através da aplicação da escala de coping, nele observa-se o percentual com maior prevalência, os de menores prevalência e aquele que não apareceu como possível estratégia de enfrentamento.

Gráfico 1: Resultado das estratégias de coping dos profissionais da saúde atuantes na linha de frente, durante a pandemia da covid-19. Echelle Toulousiane de Coping, versão (2014).

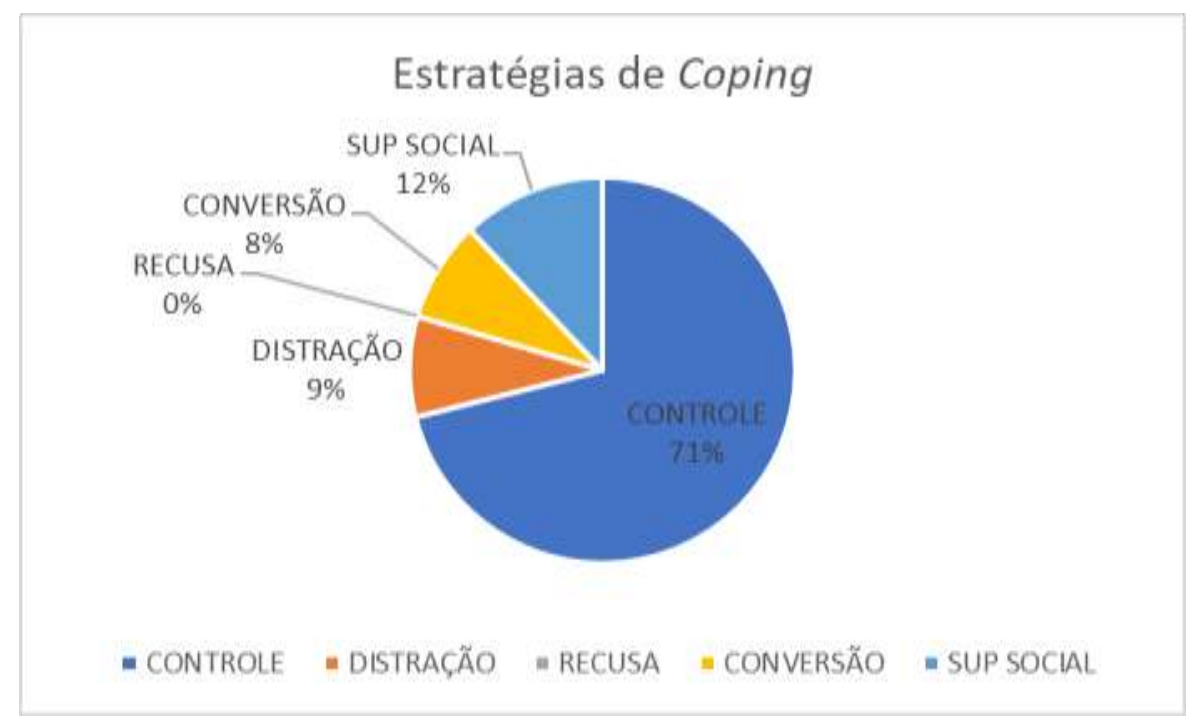

Fonte: Nunes, et al. (2014); Ribeiro, Montelo \& Soares (2021).

Entre os resultados obtidos, $71 \%$ dos participantes demonstraram como estratégia de enfrentamento o controle. Sendo considerada uma estratégia baseada no autocontrole, através da coordenação de comportamentos e na contenção de emoções, ou seja, por meio dessa, o sujeito é capaz de conter os impulsos, tornando-se reflexivo, pensando antes de agir, avaliando as situações e circunstâncias. A tomada de decisão surge após uma avaliação real da situação (Antoniazzi; Dell'aglio; Bandeira, 1998).

O Controle caracteriza-se através das reações frente ao stress, momento no qual o indivíduo reflete sobre as melhores estratégias a serem utilizadas, não evitando a confrontação (Nunes, et al., 2014). Mediante de uma situação específica na qual o ser humano desenvolve ações e reavaliações de modo cognitivo buscando enfrentamento, está se baseando numa estratégia de controle.

O resultado apurado na pesquisa vai de encontro com dados de Chamon (2006) e Pereira Et al (2016), que majoritariamente concluiu que profissionais da saúde (técnicos e enfermeiros), utilizavam o controle como estratégia de enfrentamento. Tal característica pode ser explicada por questões culturais que influenciam os profissionais da saúde na sua tomada e decisão da estratégia a ser utilizada

Em relação à estratégia de distração, $9 \%$ dos entrevistados apresentam como estratégia de enfrentamento, na qual as pessoas utilizam como recursos a companhia de outras pessoas, e o desenvolvimento de atividades que gera prazer, afim de não pensar no problema. A estratégia de distração está amplamente ligada à recusa, por aspectos de fuga da vivência (Nunes, Et al., 2014; Tap, Costa E Alves, 2005).

Entre os aspectos avaliados podemos incluir a característica de recusa, na qual o indivíduo cria um distanciamento cognitivo e emocional, por vezes age como se não houvesse o problema. Nesse caso especificamente não houve resultado como estratégia de enfrentamento. É importante levar em conta que se trata de profissionais da saúde que em momento de crise 
não puderam se ausentar de suas funções laborais. Resultados semelhantes foram encontrados em outros trabalhos (Nunes, Et al., 2014; Chamon, 2006; Maturana E Vale, 2014).

A estratégia de conversão, encontrada em $8 \%$ dos participantes, caracteriza-se pela alteração nos padrões de comportamento e pela adaptação de sua relação com problema. O indivíduo apresenta isolamento em relação aos outros e responsabilização por gerir o problema (Tap, Costa E Alves, 2005; Nunes, Et al., 2014). Segundo Chamon (2006) a estratégia de conversão implica numa mudança de atitudes, evidencia aspectos de comportamento, cognitivo ou de valores, podendo assim, gerar uma mudança de atitude, aceitação, ou a adoção de uma filosofia religiosa.

A rede de apoio em situações de crise é de fundamental importância, pois trata-se de uma ferramenta de suporte social, na qual o indivíduo se sente seguro para ser ouvido e confortado. Entre os entrevistados $12 \%$ apresentaram a rede de apoio como estratégia de enfretamento, o uso de tal estratégia sugere a necessidade de compartilhar a vivência, marcado por um momento de escuta, conforto e reconhecimento (Tap, Costa E Alves, 2005; Nunes, Et al., 2014).

A pesquisa realizada evidenciou as principais estratégias de enfrentamento de membros da equipe multiprofissional. Anteriormente, Maturana e Vale (2014) realizaram uma pesquisa similar, analisando dados de categorias específicas, como psicólogos e assistentes sociais, concluindo que as estratégias de enfrentamento mais utilizadas foram de Resolução de Problemas e Suporte Social.

\section{Conclusão}

Com base no estudo ora apresentado, conclui-se que o objetivo de identificar e descrever as estratégias de enfrentamento mais utilizadas por profissionais da saúde inseridos em um hospital referência no combate à pandemia da Covid-19, localizado no interior da Amazônia Legal foi alcançado, evidenciado que as estratégias de enfretamento mais utilizadas foram o Controle e o Suporte Social e que, as estratégias menos utilizadas, foram Recusa seguida de Conversão e Distração.

Os resultados apresentados nessa pesquisa coincidem com os dados apresentados em estudos anteriores, evidenciando uma variedade nas estratégias de enfrentamento dos profissionais de saúde diante do estresse vivenciado, fazendo se necessário repensar sobre a importância dos aspectos emocionais, inerentes a prática profissional e ao cenário de pandemia, uma vez que apesar de utilizarem-se de estratégias de enfretamento, as mesmas podem não ser suficientes para lidar com a situação, podendo assim, causar sofrimento mental e emocional.

Nesse sentido, sabemos existir uma variedade de recursos que podem ser utilizados no intuito de minimizar o sofrimento, onde citamos como possíveis, o acompanhamento psicológico e a validação da vivência, uma vez que pensar estratégias e resultados obtidos são inerentes às mudanças de rotina/comportamento. Em relação aos resultados encontrados, os dados são relevantes, validando a similaridade com pesquisas anteriores, situação e a forma de enfrentamento desta, pois além da avaliação cognitiva pessoal, o ambiente de trabalho, no caso o hospital, tem papel importante na escolha das estratégias de enfrentamento pelos profissionais inseridos nesse ambiente.

Ressalta-se a importância de utilizar os dados obtidos e aqui apresentados para pensar estratégias de intervenção frente os resultados aqui apresentados, pois é importante avaliar se a estratégia de controle, predominante nesse estudo, pode ser considerada como positiva ou se de alguma forma causa sofrimento mental ao profissional de saúde. Sugere-se como possibilidades de intervenção, a realização de treinamentos de equipes, buscando o desenvolvimento de habilidade comunicacionais, fortalecimento das equipes através do apoio mutuo, entre outras possibilidades a serem avaliadas.

Finalizando, percebe-se que, apesar de existir um aumento na produção de dados que evidenciem características regionais, esses números ainda são insuficientes para retratar uma realidade regional, sendo de vital importância novos estudos 
e produções científicas. Notam-se aspectos amplos nas estratégias utilizadas e não podemos deixar de mencionar a característica única que é viver uma experiência pandêmica.

\section{Referências}

Brasil. (2019). Ministério da Saúde. Portaria nº 188 GM/MS, de 3 de fevereiro de 2020. Declara Emergência em Saúde Pública de importância Nacional (ESPIN) em decorrência da Infecção Humana pelo novo Coronavírus http://bvsms.saude.gov.br/bvs/saudelegis/gm/2020/prt0188_04_02_2020.html

Brasil. (2020). Ministério da Saúde. Lei no 13.979, de 6 de fevereiro de 2020. DIÁRIO OFICIAL DA UNIÃO. Publicado em: 07/02/2020 Edição: 27 Atos do Poder Legislativo. https://www.in.gov.br/en/web/dou/-/lei-n-13.979-de-6-de-fevereiro-de-2020-242078735

Brasil. (2020). Ministério da Saúde. PORTARIA No 1.802, DE 20 DE JULHO DE 2020. DIÁRIO OFICIAL DA UNIÃO. Publicado em: 23/07/2020. Edição: 15, Seção 1, Página 76. Atos do Poder Legislativo. HTTPS://WWW.IN.GOV.BR/EN/WEB/DOU/-/PORTARIA-N-1.802-DE-20DE-JULHO-DE-2020-268194029

da Silva, G. A. V., da Silva, G. D. S. A., Andolhe, R., da Silva, R. M., Padilha, K. G., \& Costa, A. L. S. (2017). Estresse e coping entre profissionais de enfermagem de unidades de terapia intensiva e semi-intensiva. Revista de Enfermagem UFPE on line, 11(2), 922-931.

Creswell, J. W., \& Clark, V. L. P. (2015). Pesquisa de Métodos Mistos-: Série Métodos de Pesquisa. Penso Editora.

Dell’Aglio, D. D., \& Bandeira, D. R. (1998). O conceito de Coping: uma revisão teórica. Estudos de Psicologia.

de Oliveira Chamon, E. M. Q. (2006). Estresse e estratégias de enfrentamento: o uso da escala Toulousaine no Brasil. Revista Psicologia: Organizações e Trabalho, 6(2), 43-64.

Fernandes, G. F., \& Inocente, N. J. (2010). Estratégias Para enfrentamento (coping): um levantamento bibliográfico. XIV Encontro Latino Americano de Iniciação Científica e X Encontro Latino Americano de Pós-Graduação-Universidade do Vale do Paraíba. Vale do Paraíba: UNIVAP, 1-5.

Ferreira, A., \& Sant'ana, F. G. F. (2018). A atuação do psicólogo frente às emergências e desastres. Caderno de Graduação-Ciências Humanas e SociaisUNIT-ALAGOAS, 5(1), 99.

Franco, M. H. D. C. (2013). Saúde mental em emergências e desastres: contribuições à prática do psicólogo

Gil, A. C. (2008). Métodos e técnicas de pesquisa social. 6. ed. Ediitora Atlas SA.

Jacobi, P. R., \& Cibim, J. (2015). A necessária compreensão das consequências ampliadas de um desastre.

Lana, R. M., Coelho, F. C., Gomes, M. F. D. C., Cruz, O. G., Bastos, L. S., Villela, D. A. M., \& Codeço, C. T. (2020). Emergência do novo coronavírus (SARS-CoV-2) e o papel de uma vigilância nacional em saúde oportuna e efetiva. Cadernos de Saúde Pública, 36, e00019620.

Lazarus, R., \& Folkman, S. (1984). Stress appraisal and coping. New York: Springer

Lima, C. M. A. D. O. (2020). Informações sobre o novo coronavírus (COVID-19)

Maturana, A. P. P. M., \& Valle, T. G. M. D. (2014). Estratégias de enfrentamento e situações estressoras de profissionais no ambiente hospitalar. Psicologia hospitalar, 12(2), 02-23.

Nunes, O., Brites, R., Pires, M., \& Hipólito, J. (2014). Escala Toulousiana de Coping-reduzida. Manual técnico de utilização. Escala Toulousiana de Copingreduzida. Manual técnico de utilização.

Opas, Organização Pan-Americana de Saúde (2020). Histórico da Pandemia de COVID-19. https://www.paho.org/pt/covid19/historico-da-pandemia-covid-19

Opas, Organização Pan-Americana de Saúde (2020). Cuidados para profissionais da saúde expostos ao novo coronavírus (COVID-19) em estabelecimentos de saúde. https://iris.paho.org/bitstream/handle/10665.2/52050/OPASBRACOVID1920046_por.pdf?sequence=1\&isAllowed=y

Pereira, A. S., Shitsuka, D. M., Parreira, F. J., \& Shitsuka, R. (2018). Metodologia da pesquisa científica.

Pereira, S. D. S., Teixeira, C. A. B., Reisdorfer, E., Vieira, M. V., Gherardi-Donato, E. C. D. S., \& Cardoso, L. (2016). A relação entre estressores ocupacionais e estratégias de enfrentamento em profissionais de nivel tecnico de Enfermagem. Texto \& Contexto-Enfermagem, 25.

Santeiro, T. V., Yoshida, E. M. P., Peixoto, E. M., Rocha, G. M. A. D., \& Zanini, D. S. (2016). Diferenças conceituais e empíricas entre eficácia adaptativa e coping. Estudos Interdisciplinares em Psicologia, 7(1), 02-19.

Tap, P., Costa, E. S., \& Alves, M. N. (2005). Escala Toulousiana de Coping (ETC): Estudo de adaptação à população portuguesa. Psicologia, Saúde \& Doenças, 6(1), 47-56.

Teixeira, C. F. D. S., Soares, C. M., Souza, E. A., Lisboa, E. S., Pinto, I. C. D. M., Andrade, L. R. D., \& Espiridião, M. A. (2020). A saúde dos profissionais de saúde no enfrentamento da pandemia de Covid-19. Ciência \& Saúde Coletiva, 25, 3465-3474. 